\title{
$\beta$-Hydroxybutyrate impairs the release of bovine neutrophil extracellular traps through inhibiting phosphoinositide 3-kinase-mediated nicotinamide adenine dinucleotide phosphate oxidase reactive oxygen species production
}

\author{
Siyuan Liu, ${ }^{1 *}$ Xiaobing Li, ${ }^{2 *}$ Xiaohan Zhou, ${ }^{1}$ Juan J. Loor, ${ }^{3}$ Qianming Jiang, ${ }^{3}$ Xiancheng Feng, ${ }^{1}$ Yuchen Yang, ${ }^{1}$ \\ Lin Lei, ${ }^{1}$ Xiliang Du, ${ }^{1}$ Xinwei Li, ${ }^{1}$ Wang Zhe, ${ }^{1}$ Yuxiang Song, ${ }^{1} \dagger$ and Guowen Liu ${ }^{1} \dagger$ \\ ${ }^{1}$ State Key Laboratory for Zoonotic Diseases, Ministry of Education, College of Veterinary Medicine, Jilin University, 5333 Xi'an Road, Changchun, \\ Jilin Province, 130062, China \\ ${ }^{2}$ College of Veterinary Medicine, Yunnan Agricultural University, Kunming 650201, China \\ ${ }^{3}$ Mammalian NutriPhysioGenomics, Department of Animal Sciences and Division of Nutritional Sciences, University of Illinois, Urbana 61801
}

\begin{abstract}
Ketosis in dairy cows often occurs in the peripartal period and is accompanied by immune dysfunction. High concentrations of $\beta$-hydroxybutyrate (BHB) in peripheral blood during ketosis inhibits the release of neutrophil extracellular traps (NET) and contributes to immune dysfunction. However, the mechanisms whereby BHB affects NET release remains unclear. In this study, 5 healthy peripartal dairy cows (within 3 wk postpartum) with serum BHB concentrations $<0.6$ $\mathrm{m} M$ and glucose concentrations $>3.5 \mathrm{~m} M$ were used as blood donors. Blood samples were collected before feeding, and the isolated polymorphonuclear neutrophils were incubated with $3 \mathrm{~m} M$ BHB for different times. Inhibition of Cit-H3 (citrullinated histone 3) protein abundance, a marker of NET activation, in response to BHB was used to determine an optimal incubation time for in vitro experiments. Four hours was selected as the optimal duration of BHB treatment. Phorbol12-myristate-13-acetate (PMA) was used to induce the release of NET in vitro. The BHB treatment with or without PMA treatment decreased protein abundance of Cit-H3 and PAD4 (arginine deiminase 4) and increased neutrophil elastase. Immunofluorescence and scanning electron microscope analyses revealed that BHB treatment inhibited PMA-induced NET release. The BHB treatment also decreased double strain DNA content in the supernatant, further confirming the inhibitory effect of BHB on NET release. Furthermore, BHB treatment decreased the level of intracellular
\end{abstract}

Received August 18, 2021.

Accepted December 16, 2021.

*These authors contributed equally to this work.

†Corresponding authors: songyuxiang2018@126.com and liuguowen2008@163.com reactive oxygen species (ROS), phosphorylation level of $\mathrm{p} 47$, and protein abundance of Rac2, suggesting that BHB-induced NET inhibition may have been caused by decreased NADPH oxidase-derived ROS. The phosphorylation level of phosphoinositide 3-kinase (PI3K), an important upstream regulator of NADPH oxidase, was attenuated by BHB treatment. To confirm the involvement of PI3K signaling pathway in BHB-induced NET inhibition, 740Y-P, a potent activator of PI3K signaling pathway, was used. Data indicated that $740 \mathrm{Y}-$ $\mathrm{P}$ relieved the inhibitory effects of BHB on ROS production and NADPH oxidase activation. Importantly, as revealed by immunofluorescence and scanning electron microscopy analyses, 740Y-P also dampened the inhibitory effect of BHB on NET release and the protein abundance of Cit-H3 and PAD4. Overall, the present study revealed that high concentration of BHB impairs NET release through inhibiting PI3K-mediated NADPH oxidase ROS production. These findings help partly explain the immune dysfunction in cows experiencing negative energy balance or ketosis in early lactation.

Key words: ketosis, neutrophil extracellular trap, nicotinamide adenine dinucleotide phosphate, reactive oxygen species, phosphoinositide 3-kinase

\section{INTRODUCTION}

Peripartal dairy cows are often in a state of negative energy balance (NEB) due to the increase in energy demands and the decrease of DMI (Lopreiato et al., 2020; Bucktrout et al., 2021; Lopes et al., 2021). Severe NEB leads to metabolic disorders including ketosis. Subclinical ketosis can be defined as a BHB concentration of 1.2 to $3 \mathrm{mM}$, and clinical ketosis can be defined as $>3 \mathrm{mM}$ (Dervishi et al., 2016). The high incidence of infections, such as mastitis, in ketotic cows has been ascribed to a state of immune dysfunction (Grinberg et 
al., 2008). In PMN studies of human, bovine, and ovine, concentrations of BHB similar to those observed during ketosis impaired the release of neutrophil extracellular traps (NET). These are web-like structures composed of chromosomal DNA associated with nuclear histone and granular antimicrobial proteins such as myeloperoxidase and neutrophil elastase (NE), which can trap and kill pathogens (Grinberg et al., 2008; Song et al., 2016; Tackenberg et al., 2021). A previous in vitro study demonstrated that high concentrations of BHB could strongly inhibit NET formation, thus providing a novel explanation for the immune dysfunction in ketotic cows (Tackenberg et al., 2021). Mechanisms whereby BHB inhibits the formation of NET are unclear.

Reactive oxygen species (ROS) in PMN are mainly produced by NADPH oxidase (Lam et al., 2010; Song et al., 2020). In phagocytes, NADPH oxidase-derived ROS are not only involved in killing of invading pathogens in phagosomes, but also act as modulators of other cell functions such as phagocytosis (Bréchard et al., 2013). Of note, in human PMN, production of ROS was crucial for NET release through promoting PAD4 (protein arginine deiminase 4)-mediated histone citrullination (Grob et al., 2020). However, whether NADPH oxidase-derived ROS is involved in the regulation of NET release in dairy cows remains unclear.

The NADPH oxidase enzyme exists largely in the form of a complex containing 2 membrane-bound polypeptides including p22 (phox) and gp91 (phox), and 4 cytosolic proteins including p47 (phox), p67 (phox), p40 (phox), and a GTPase Rac2 (El-Benna et al., 2009). The enzyme is mainly activated by phosphorylation of p47, promoting its binding with p22 and Rac2 binding with gp91 (El-Benna et al., 2009; Bréchard et al., 2013). Studies in bovine PMN have shown that ROS production in PMN is reduced by exposure to high concentrations of BHB in vitro (Grinberg et al., 2008). Thus, assessing the role of NADPH oxidase-derived ROS in the BHB-induced inhibition of NET release in PMN of dairy cows merits further study.

Phosphoinositide 3-kinase (PI3K) is a family of lipid kinases whose activation in human PMN generates PIP3 and promotes formation of the Rac2 and gp91 complex that leads to p47 phosphorylation (Song et al., 2020). Studies have shown that high concentrations of BHB inhibit activation of the PI3K signaling pathway in bovine hepatocytes (Angeli et al., 2021). These previous findings suggest that the PI3K-NADPH oxidase-ROS signaling pathway may be involved in the regulation of NET. Thus, we surmise that BHB decreases NADPH oxidase-derived ROS by inhibiting the PI3K signaling pathway, thereby impairing the release of NET. Our objective was to explore the effect of BHB on the release of NET and the underlying molecular mechanisms.

\section{MATERIALS AND METHODS}

\section{Animals}

The Ethics Committee on the Use and Care of Animals at Jilin University approved the present experimental protocol (no. 202012017). Animals used in the current study received humane care according to the principles and guidelines of the Guide for the Care and Use of Agricultural Animals in Research and Teaching (FASS, 2010). The selection of experimental animals was similar to previous studies (Song et al., 2016; Du et al., 2018b). In brief, healthy dairy cows in the peripartal period (within 3 wk postpartum) with similar parities, BW, and milk yield were preselected from a dairy farm located in Changchun City (Jilin province, China). In addition, to ensure that the circulating PMN were in a low BHB environment before sampling, we selected postpartum cows in a low degree of NEB as PMN donors. In brief, 15 healthy cows with negative nitroprusside test in milk were preselected. Among these cows, 5 cows in a less pronounced NEB state (serum BHB concentrations $<0.6 \mathrm{~m} M$ and serum glucose concentrations $>3.5 \mathrm{mM}$ ) were finally selected in the present study (Itle et al., 2015; Song et al., 2021). Serum samples were collected in the morning (0800 h) for 3 consecutive days.

All selected cows were housed in a climate-controlled barn with individual tiestalls to reduce environmental interference. Cows had ad libitum access to the same diet (Du et al., 2018a) that was offered twice daily (0830 and $1600 \mathrm{~h}$ ), and fresh water was supplied continuously.

\section{Isolation, Culture, and Treatment of PMN}

Blood was collected from the jugular vein of dairy cows in the morning for 3 consecutive days (0800 h) using a vacutainer blood collection tube with trisodium citrate and kept at room temperature until transport to the laboratory (within 30 min after collection) for subsequent PMN isolation. The PMN were isolated using a commercial bovine peripheral blood PMN isolation kit (cat. no. P9400; Solarbio Science and Technology Co. Ltd.) according to the manufacturer's instructions. In brief, PMN were separated based on density gradient centrifugation. Erythrocyte lysis buffer (cat. no. R1010; Solarbio) was added to remove red blood cells followed by washing the remaining cells twice with RPMI-1640 basic medium (cat. no. SH30809.01; Hyclone Laboratories) and centrifuging for $10 \mathrm{~min}$ at $500 \times \mathrm{g}$ to har- 
vest PMN. The separation system was kept at room temperature throughout the experiment to eliminate the influence of temperature change on the activity of PMN. Isolated PMN were identified by Giemsa (cat. no. G1020; Solarbio) and trypan blue staining (cat. no. C0040; Solarbio). Purity of the obtained PMN was $\sim 97.5 \%$ with $\sim 95 \%$ viability.

Cells were resuspended in basal culture medium, which contained $10 \%$ fetal bovine serum (Hyclone Laboratories) and 1\% penicillin-streptomycin (cat. no. Sv30010; Hyclone) in RPMI medium (cat. no. SH30809.01, Hyclone Laboratories). After cell counting, the cell suspension was adjusted to $1 \times 10^{6}$ cells $/ \mathrm{mL}$ and seeded in 6 -well ( $3 \mathrm{~mL}$ per well) and 24 -well culture plates $(1 \mathrm{~mL}$ per well). The PMN were then incubated at $37^{\circ} \mathrm{C}$ for $1 \mathrm{~h}$ in a cell incubator with humidified atmosphere and $5 \% \mathrm{CO}_{2}$ followed by $\mathrm{BHB}$ treatment.

Concentrations of BHB (cat. no. 55397; SigmaAldrich) used in our study were selected according to normal and pathological hematology standards for dairy cows with or without ketosis (Zhu et al., 2019). The BHB powder was dissolved in PBS and sterilized by filtration. The PMN were treated with $3 \mathrm{~m} M \mathrm{BHB}$ for $0,2,4,6$, and $8 \mathrm{~h}$. The BHB treatment for $4 \mathrm{~h}$ had an obvious inhibition effect on the citrullination of histone, and was selected as the incubation time for subsequent experiments. To verify the role of the PI3K signaling pathway in NET release, the PI3K signaling activator 740Y-P (10 $\mu M$, cat. no. HY-P0175; MCE) was preincubated for $0.5 \mathrm{~h}$ before BHB treatment. Phorbol-12-myristate-13-acetate (PMA; $100 \mathrm{ng} / \mathrm{mL}$, cat. no. P8139, Sigma-Aldrich) was incubated for $3 \mathrm{~h}$ to induce NET.

\section{Western Blot Analysis}

Total protein in PMN was extracted according to manufacturer's instructions (Sangon Biotech Co. Ltd.). Protein concentration was measured by the bicinchoninic acid method (cat. no. P1511; Applygen Technologies). A total of $30 \mu \mathrm{g}$ of protein from each sample was separated by 8 to $15 \%$ SDS-PAGE and electro-transferred onto polyvinylidene difluoride (PVDF) membranes at $80 \mathrm{~V}$ for $45 \mathrm{~min}$. Then, PVDF membranes were blocked with $3 \%$ BSA in Tris-buffered saline with $0.1 \%$ Tween-20 detergent for $4 \mathrm{~h}$ at room temperature and hybridized with primary specific antibodies overnight at $4^{\circ} \mathrm{C}$, including p47 (1:1,000; cat. no. AF5220; Affinity Biosciences), phosphorylated-p47 (1:1,000; YP1018; Immunoway Biotechnology), Rac2 (1:1,000; cat. no. YN1162; Immunoway Biotechnology), NE (1:1,000; cat. no. YN1936; Immunoway Biotechnology), Cit-H3 (1:1,000; cat. no. ab5103; Abcam), PAD4 (1:1,000; cat. no. ab214810; Abcam), phosphorylated-
PI3K (1:1,000; cat. no. bs-6417R; Bioss), PI3K (1:1,000; cat. no. bs-10657R; Bioss), and $\beta$-actin (1:2,000; cat. no. ab8226; Abcam). The PVDF membranes were then washed with Tris-buffered saline with $0.1 \%$ Tween-20 detergent for $3 \times 5$ min and incubated with horseradish peroxidase-conjugated anti-rabbit (1:5,000; cat. no. SA00001-2; ProteinTech Group, Inc.) or anti-mouse (1:5,000; cat. no. SA00001-1; ProteinTech Group Inc.) immunoglobulin for $1 \mathrm{~h}$ at room temperature. Immunoreactive bands were detected with an enhanced chemiluminescence solution (cat. no. WBKLS0500; Millipore). $\beta$-actin was used as a reference protein. Last, all bands were imaged using a Protein Simple Imager (ProteinSimple), and their gray value was analyzed using the Image-pro Plus (Media Cybernetics). Western blots were run in triplicate for each experimental group.

\section{Cell Viability Assay}

Cell viability of PMN was analyzed by a cell counting kit-8 (CCK-8, cat. no. 176 CK04-11; Dojindo Molecular Technologies) according to the manufacturer's instructions. In brief, $1 \times 10^{5} \mathrm{PMN}(100 \mu \mathrm{L} /$ well $)$ were seeded into the 96 -well plates and were exposed to $3.0 \mathrm{mM}$ BHB for $0,2,4,6$, and $8 \mathrm{~h}$. After treatment, CCK-8 solution $(10 \mu \mathrm{L})$ was added and incubated for $1 \mathrm{~h}$ in a cell incubator with humidified atmosphere and $5 \% \mathrm{CO}_{2}$ at $37^{\circ} \mathrm{C}$. Finally, the absorbance at $450 \mathrm{~nm}$ was measured with a Multiskan FC microplate reader (cat. no. 51119180ET; Thermo Fisher Scientific). Cell viability was calculated by (average absorbance value of experimental group/average absorbance value of control group) $\times 100 \%$.

\section{Immunofluorescence Assay}

The PMN $\left(1 \times 10^{6} / \mathrm{mL}\right)$ were attached to laser confocal dishes (cat. no. 801002; NEST) coated with 0.1 $\mathrm{mg} / \mathrm{mL}$ poly-D-lysine (cat. no. P1149; Sigma-Aldrich). After treatment, PMN were washed twice with PBS and then fixed with $4 \%$ paraformaldehyde for $20 \mathrm{~min}$. The PMN were permeabilized using $0.1 \%$ Triton X-100 (Beyotime Biotechnology Inc.) and then incubated in Block buffer (1\% BSA, $10 \%$ goat serum, $0.1 \%$ Triton $\mathrm{X}-100 / \mathrm{PBS}$ ) for $1 \mathrm{~h}$ at room temperature to minimize nonspecific antibody binding. Subsequently, PMN were incubated with specific antibodies overnight at $4^{\circ} \mathrm{C}$. Then, PMN were incubated with secondary antibodies for $1 \mathrm{~h}$ at room temperature. The PMN nucleus was stained by DAPI (Beyotime Biotechnology Inc.) for $20 \mathrm{~min}$. Last, coverslips were sealed with glycerol and samples imaged using laser confocal microscopy (FV500, Olympus). The extracellular co-localization of DNA and histone was regarded as the hallmark of NET 
in the present study. The immunofluorescence experiments were repeated at least 5 times; each time, at least 10 watching zones were selected for evaluation, and at least 500 cells were calculated in each group. Ratio of NET cells $=$ number of NET releasing cells/number of total cells.

\section{Scanning Electron Microscopy Analysis}

The 14-mm round cover glasses were sterilized and coated with poly-D-lysine. The coated cover glasses were placed in the 24-well cell culture plate and PMN $\left(1 \times 10^{6} / \mathrm{mL}\right)$ seeded on the prepared cover glass. The process of cell treatment was similar to the above. After incubation, cells were washed twice with PBS and then fixed with $4 \%$ glutaraldehyde for $24 \mathrm{~h}$. Cells were washed twice with PBS, fixed with $0.5 \%$ osmium tetroxide again, and washed 3 times with PBS. Samples were dehydrated in an ascending alcohol series (30, 40, $50,60,70,80,90$, and $100 \%$ ) for $2 \mathrm{~min}$ each, and subsequently sputtered with gold. Morphological characteristics of PMN were observed using a scanning electron microscopy (S-3400N, Hitachi).

\section{Quantification of NET}

We analyzed NET quantitatively by detecting extracellular double strain (ds)DNA using the PicoGreen dsDNA assay kit (cat. no. MF0781; MKBio) according to manufacturer's instructions. The PMN $\left(1 \times 10^{6} / \mathrm{mL}\right)$ were seeded into 24-well plates in RPMI-1640 (phenolred-free). The process of cell treatment was similar to the descriptions in the previous section of the paper. After incubation, $100 \mu \mathrm{L}$ of supernatant and $100 \mu \mathrm{L}$ of PicoGreen working solution were mixed in the 96-well plate, avoiding light for $5 \mathrm{~min}$ at room temperature. Fluorescence intensity was then measured at an excitation wavelength of $480 \mathrm{~nm}$ and an emission wavelength of $520 \mathrm{~nm}$ by an infinite M200 pro fluorescence microplate reader (Tecan).

\section{Determination of Intracellular ROS Concentration}

The PMN were harvested when incubated with 3 $\mathrm{m} M$ BHB (with or without $10 \mu M 740 \mathrm{Y}-\mathrm{P}$ ) for $4 \mathrm{~h}$. After treatment, PMN were incubated with dichlorofluorescin diacetate (Beyotime Biotechnology Inc.) for $30 \mathrm{~min}$ at $37^{\circ} \mathrm{C}$. The PMN were collected by centrifugation and washed 3 times with cold PBS, followed by resuspension by $200 \mu \mathrm{L}$ of PBS. For determination of ROS content, the fluorescence intensity of PMN was measured by flow cytometry (Becton Dickinson).

\section{Statistical Analysis}

A 2-way ANOVA was performed when there were 2 treatment factors (BHB and 740Y-P; BHB and PMA), including a Tukey analysis when significant interactions occurred. One-way ANOVA with subsequent Bonferroni correction or independent-samples $t$-tests was used when there was only 1 treatment factor. Other nonnormally distributed data were analyzed with Wilcoxon signed-rank test. Linear and quadratic contrasts were conducted to evaluate time-dependent effects. All analyses were performed using GraphPad Prism 8.0 (Graph Pad Software) or SPSS 19.0 software (IBM). Results are expressed as means \pm standard error of the means. $P<0.05$ was considered significant, and $P<0.01$ were considered markedly significant. All experiments were repeated at least 3 times.

\section{RESULTS}

\section{BHB Inhibited the Release of PMA-induced Extracellular Traps in Bovine PMN}

The protein abundance of Cit-H3, a marker protein of NET, was decreased due to increased duration of incubation with BHB $(P<0.01$, Figure $1 \mathrm{~A}$ and $\mathrm{B})$ with a linear $(P<0.01)$ and quadratic $(P<0.01)$ effect. Cit$\mathrm{H} 3$ protein abundance decreased significantly after $4 \mathrm{~h}$ of BHB treatment $(P<0.01$, Figure $1 \mathrm{~A}$ and $\mathrm{B})$. The $3.0 \mathrm{~m} M$ BHB treatment for 0 to $8 \mathrm{~h}$ had no significant effect on cell viability (Figure 1C). Compared with the control group (sterile PBS), PMA treatment increased protein abundance of Cit-H3 and PAD4 $(P<0.01)$ and it decreased NE $(P<0.01)$; BHB treatment inhibited the effects of PMA on these markers of NET $(P<0.01)$ (Figure 1D-G).

Compared with the control group, PMA treatment increased the ratio of bovine NET cells $(P<0.01$; Figure 2A). However, BHB treatment inhibited the PMAinduced ratio of NET cells $(P<0.01$; Figure $2 \mathrm{~A})$. The scanning electron microscopy data further confirmed the above phenotypes (Figure 2B). The dsDNA in cell culture supernatant data showed that PMA treatment increased the level of dsDNA, whereas BHB inhibited this effect $(P<0.01$; Figure $2 \mathrm{C})$.

\section{BHB Inhibited NADPH Oxidase-derived ROS Production in Bovine PMN}

Compared with the control group, BHB treatment reduced intracellular ROS levels in bovine PMN $(P<$ 0.01; Figure 3A and B). In addition, BHB treatment reduced the phosphorylation of $\mathrm{p} 47(P<0.01$; Figure 
A

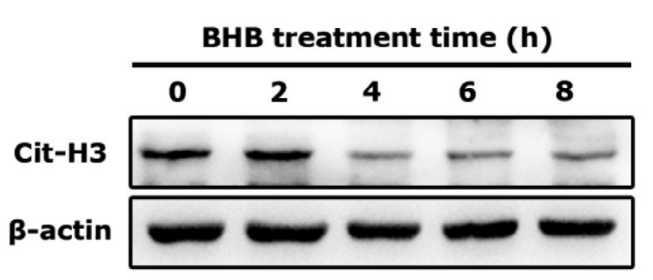

B

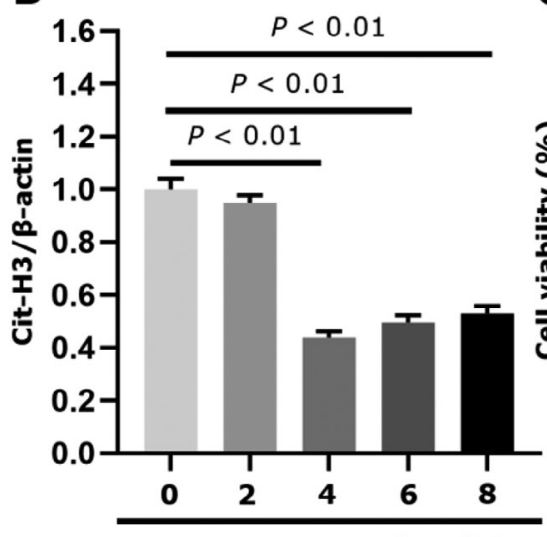

BHB treatment time (h)
C

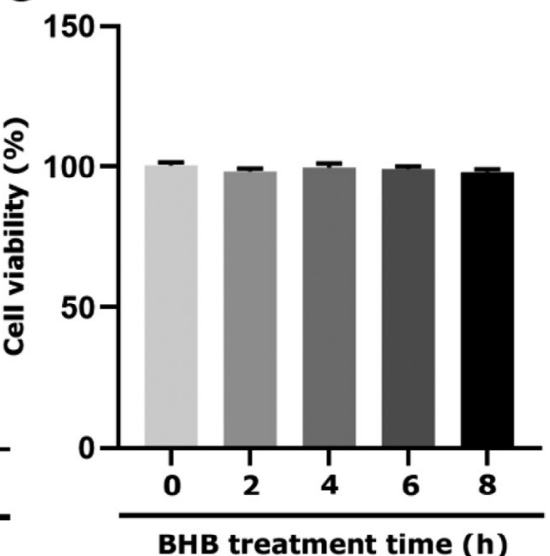

D

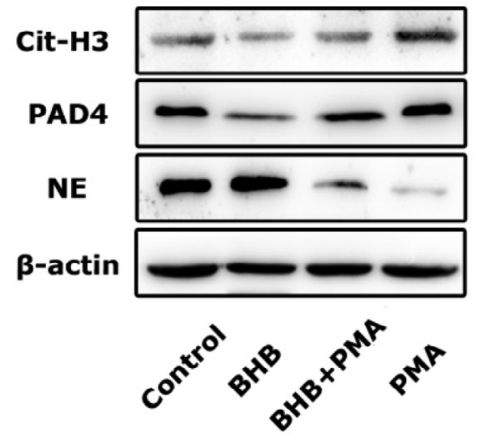

G

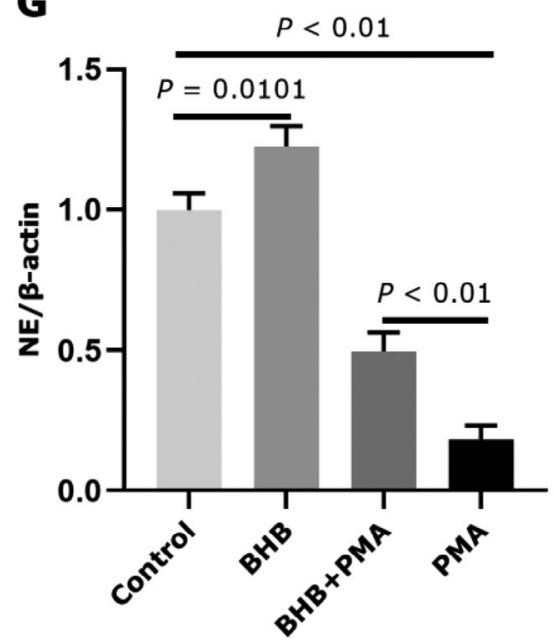

E

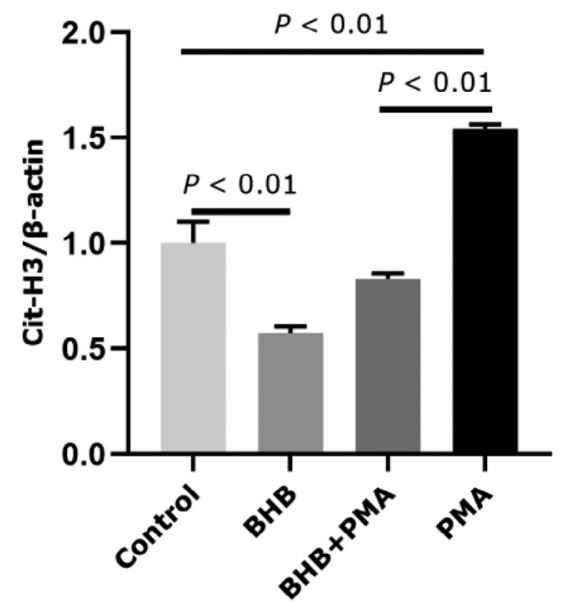

$\mathbf{F}$

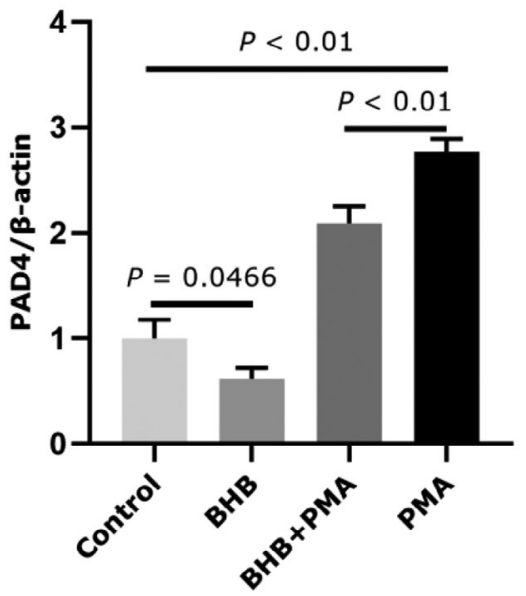

Figure 1. Effects of BHB on the expression of bovine neutrophil extracellular trap (NET) markers. (A, B) Protein abundance of citrullinated histone 3 (Cit-H3) at different times after BHB treatment $(3 \mathrm{mM})$. Representative blots in both groups are shown in panel A, and the quantification in panel B. We selected $3 \mathrm{~m} M$ BHB for $4 \mathrm{~h}$ for subsequent experiments. (C) The effect of different times of BHB (3.0 m $M$ ) on the cell viability. (D-G) Protein abundance of Cit-H3, PAD4, and NE after BHB treatment. We used $100 \mathrm{ng} / \mathrm{mL}$ PMA treatment for $3 \mathrm{~h}$ to induce NET release. Representative blots in both groups are shown in panel C, and the quantification in panels D-F. $\beta$-Actin was used to normalized protein abundance. Two-way ANOVA revealed a BHB $\times$ PMA interaction for Cit-H3 $(P<0.05)$. Experiments were repeated 5 times. Data were analyzed with 1-way or 2-way ANOVA. All data are expressed as means \pm SEM. A $P<0.05$ was deemed significant and $P<0.01$ highly significant. PMA = phorbol-12-myristate-13-acetate; PAD4 = protein arginine deiminase 4; $\mathrm{NE}=$ neutrophil elastase. 
A
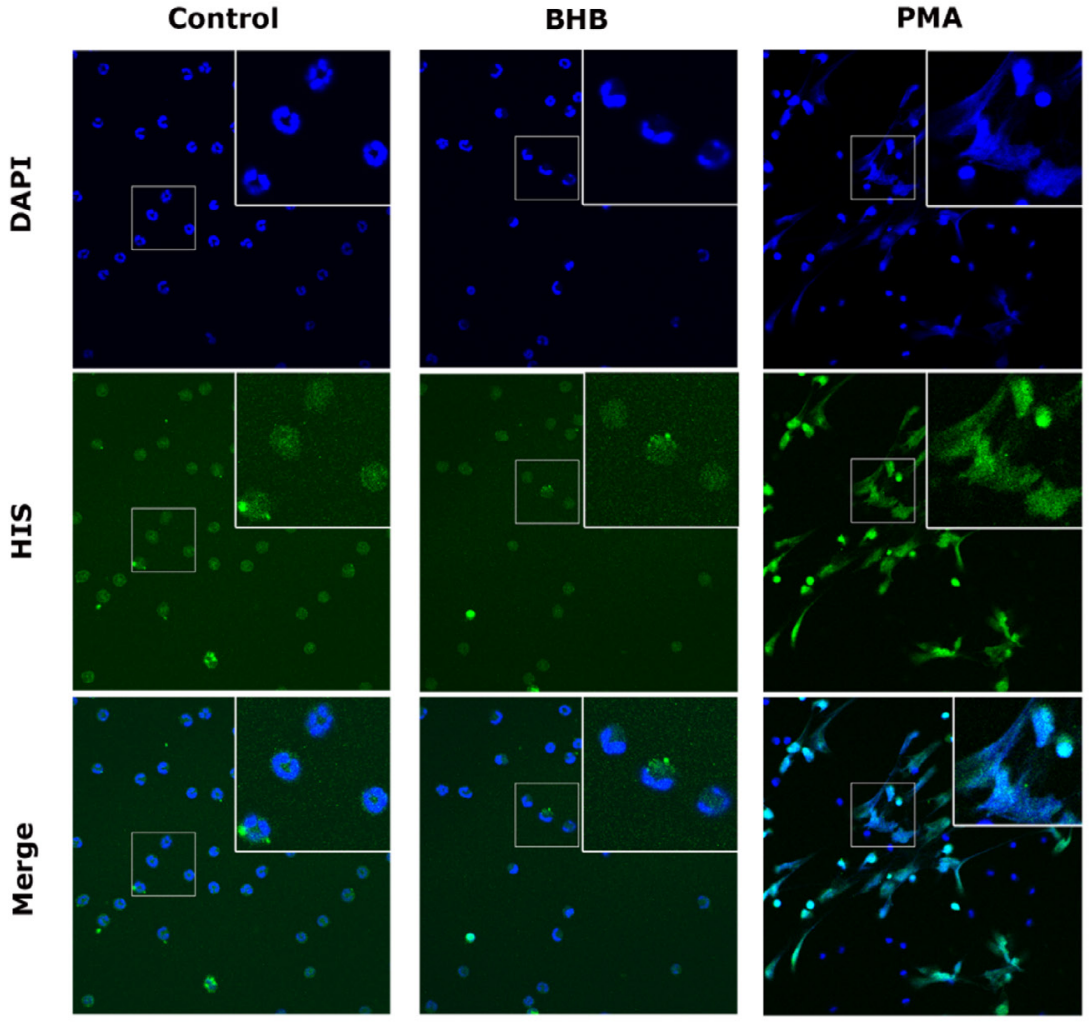

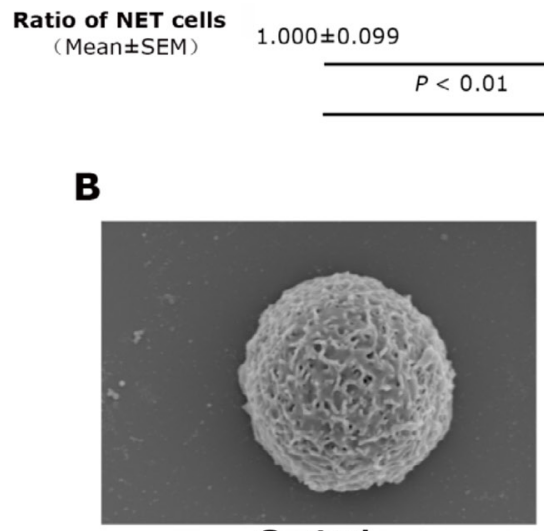

Control

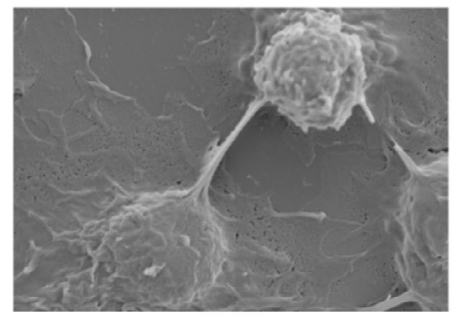

PMA
$0.532 \pm 0.014$

$P<0.01$

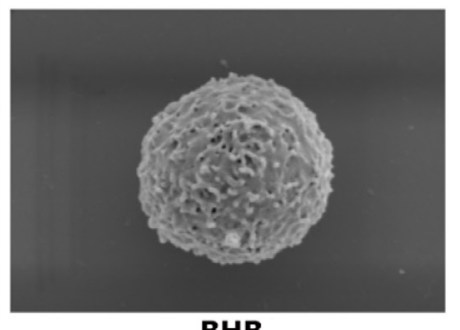

BHB

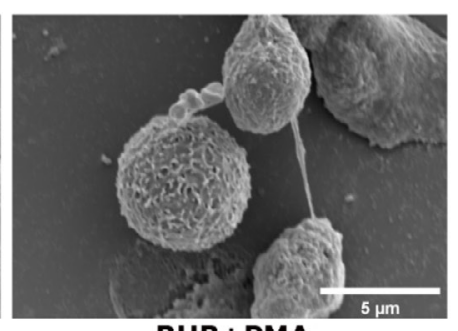

BHB+PMA

$18.529 \pm 0.373$

BHB+PMA
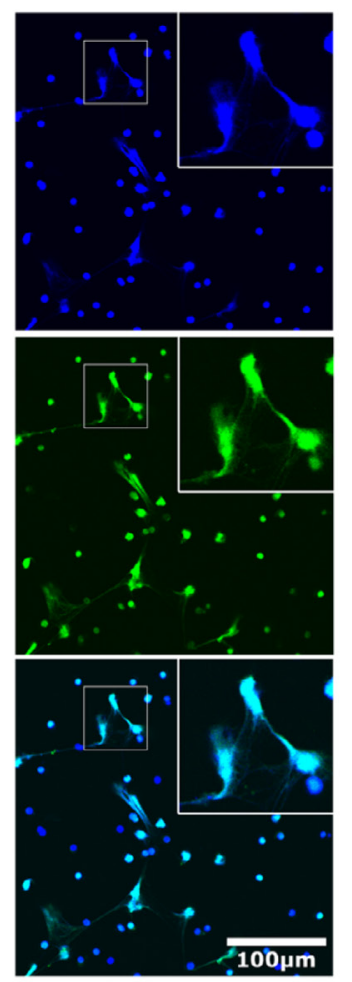

$P<0.01$
$9.720 \pm 0.218$

\section{C}

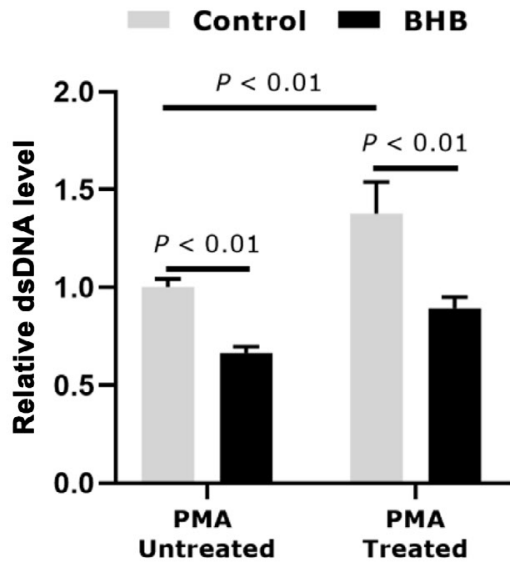

Figure 2. Effects of BHB on the release of bovine neutrophil extracellular traps (NET). (A) Immunofluorescence experiments demonstrated the release of NET structures from PMN. The PMA $(100 \mathrm{ng} / \mathrm{mL})$ treatment for $3 \mathrm{~h}$ was used to induce NET formation. Magnification $=400 \times$. Scale bar $=100 \mu \mathrm{m}$. Nuclei/DAPI is in blue, and HIS is in green. Images represent the typical common phenotype for each group. Ratio of NET cells is shown under the image. At least 500 cells were calculated in each group. Two-way ANOVA revealed a BHB $\times$ PMA interaction $(P<$ 0.05). (B) Scanning electron microscopy analysis of NET. Magnification $=10,000 \times$. Scale bar $=5 \mu$ m. (C) The double strain DNA (dsDNA) levels in the supernatant. Experiments were repeated 5 times. Data are expressed as means \pm SEM. A $P<0.05$ was deemed significant and $P$ $<0.01$ highly significant. PMA= phorbol-12-myristate-13-acetate; HIS = histone H3. 
A

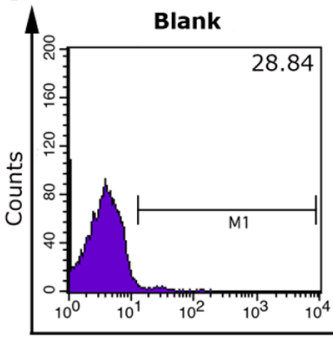

C

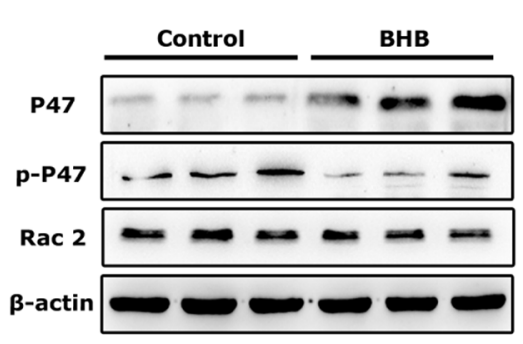

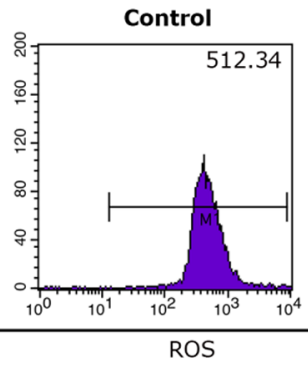

D

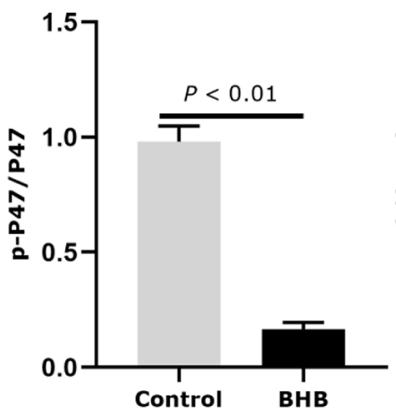

B

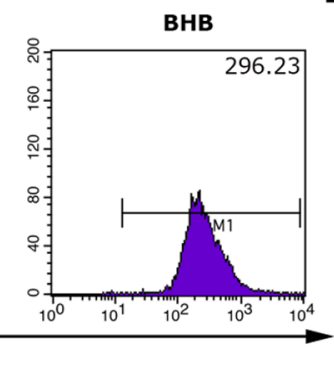

E
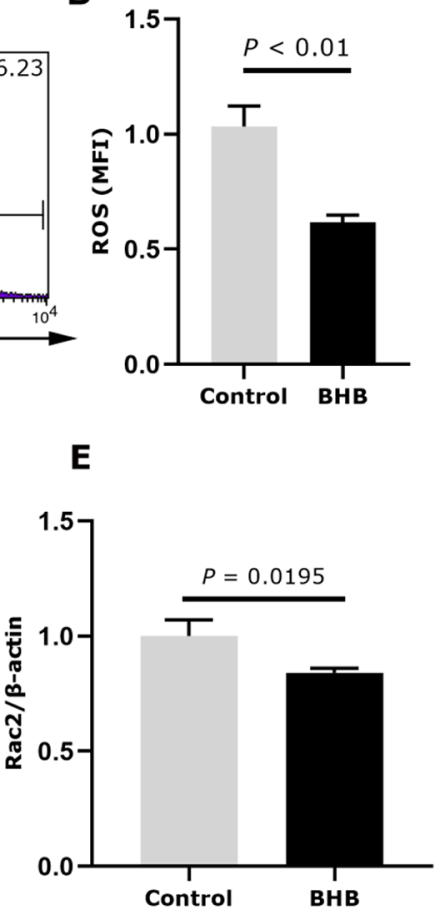

Figure 3. Effects of BHB on the NADPH oxidase-derived reactive oxygen species (ROS) production. (A) Flow cytometry analysis of intracellular ROS. Mean fluorescence intensity values of representative graphs for each group are shown in the upper right corner. (B) Quantitation of ROS levels in panel A. (C-E) Protein abundance of p47, phosphorylated (p)-p47, and Rac2. Representative blots for both groups (3 wells of each group represent 3 samples from different cows, respectively) are shown in panel C, and quantification in panel D and E. $\beta$-actin was used to normalize protein abundance. The above experiments were repeated 5 times. All data are expressed as means \pm SEM. A $P<0.05$ was deemed significant and $P<0.01$ highly significant. MFI $=$ mean fluorescence intensity.

$3 \mathrm{C}$ and $\mathrm{D})$ and the protein abundance of $\operatorname{Rac} 2(P=$ 0.0195 ; Figure $3 \mathrm{E})$.

\section{Role of PI3K Signaling Pathway in the BHB-induced Inhibition of NADPH Oxidase ROS Production in Bovine PMN}

Compared with the control group, BHB treatment decreased the phosphorylation of PI3K $(P<0.01$; Figure $4 \mathrm{~A}$ and $\mathrm{B})$. $740 \mathrm{Y}-\mathrm{P}(10 \mu M, 4.5 \mathrm{~h})$, an activator of PI3K, increased phosphorylation of PI3K and inhibited this effect of BHB $(P<0.01$; Figure $4 \mathrm{C}$ and $\mathrm{D})$. The 740Y-P treatment increased phosphorylation of p47 ( $P$ $<0.01)$ and the protein abundance of $\operatorname{Rac} 2(P<0.01)$, and inhibited this effect of BHB $(P<0.01)$ (Figure $4 \mathrm{C}$, $\mathrm{E}$, and $\mathrm{F})$. The $740 \mathrm{Y}-\mathrm{P}$ treatment enhanced production of ROS $(P<0.01)$, and reduced this effect of BHB ( $P$ $<0.01$; Figure $4 \mathrm{G}$ and $\mathrm{H})$.

\section{PI3K Signaling Mediates the Inhibitory Effect of BHB on NET in Bovine PMN}

As detected by immunofluorescence experiments, compared with the PMA only group, additional 740Y-
$\mathrm{P}$ treatment increased the release of NET $(P<0.01$; Figure $5 \mathrm{~A})$, and reversed this effect of $\mathrm{BHB}(P<0.01$; Figure $5 \mathrm{~A})$. The $740 \mathrm{Y}-\mathrm{P}$ treatment enhanced the protein abundance of Cit-H3 and PAD4 $(P<0.01)$, and inhibited this effect of BHB $(P<0.01$; Figure $5 \mathrm{~B}-\mathrm{D})$.

\section{DISCUSSION}

Clinical concentrations of blood BHB are associated with dysfunction of the innate immune system in peripartal dairy cows, thereby increasing the susceptibility of mastitis and endometritis (Galvão et al., 2010; Dervishi et al., 2016). The PMN are the main effector cells in the innate immune system and act as the first line of defense against pathogens infection (Borregaard, 2010; Galvão et al., 2010). In humans, PMN function such as phagocytosis was impaired by clinical concentration of BHB (Wilson and Reeves, 1986). Furthermore, clinical concentrations of blood $\mathrm{BHB}$ were reported to negatively affect the formation of NET in bovine PMN challenged with bacteria (Grinberg et al., 2008). The PMA induces NET formation strongly by activating NADPH oxidase and ROS production (Burgener and Schroder, 2020). In the present study, we also demon- 
A

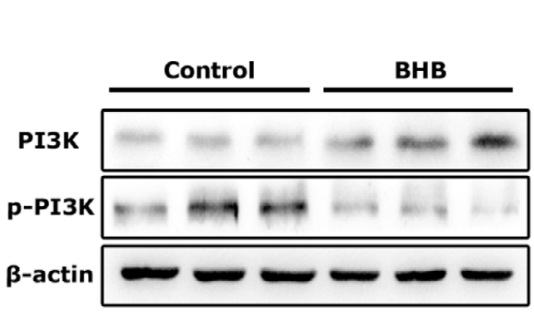

B

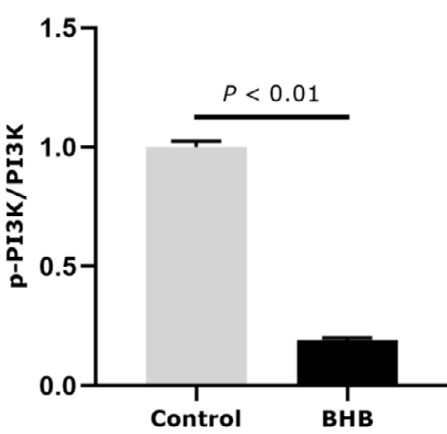

C

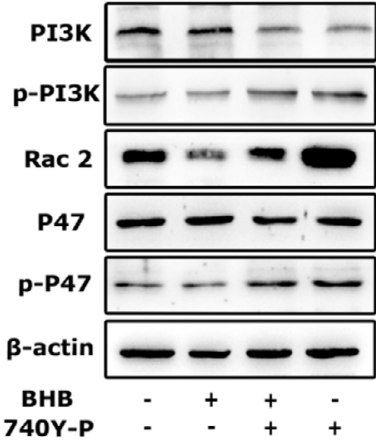

D

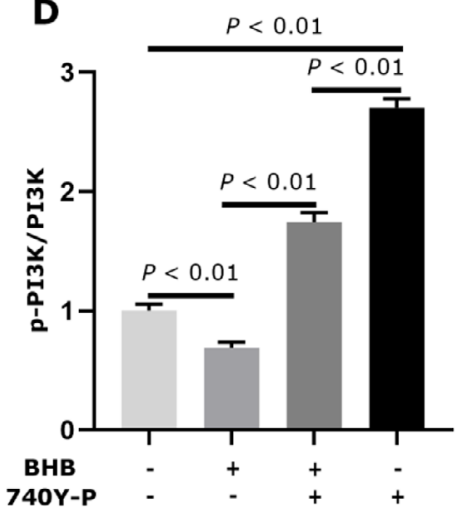

E

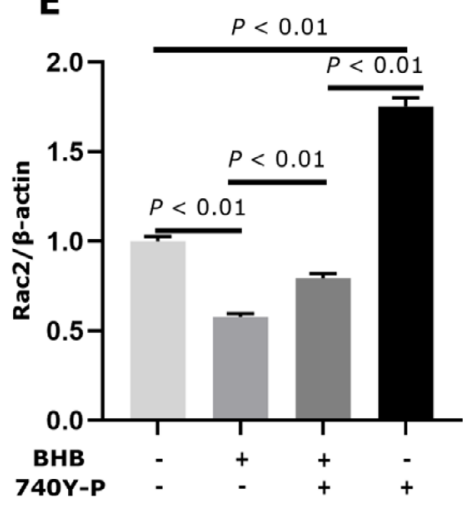

$F$

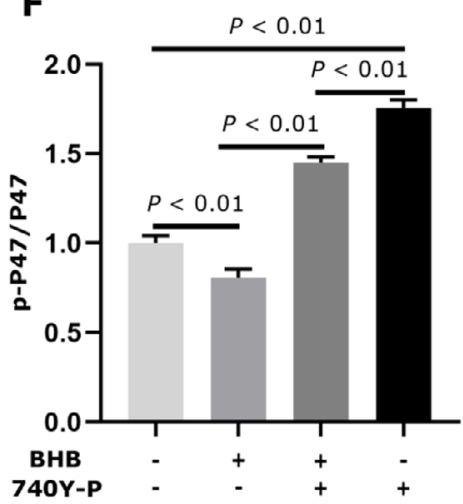

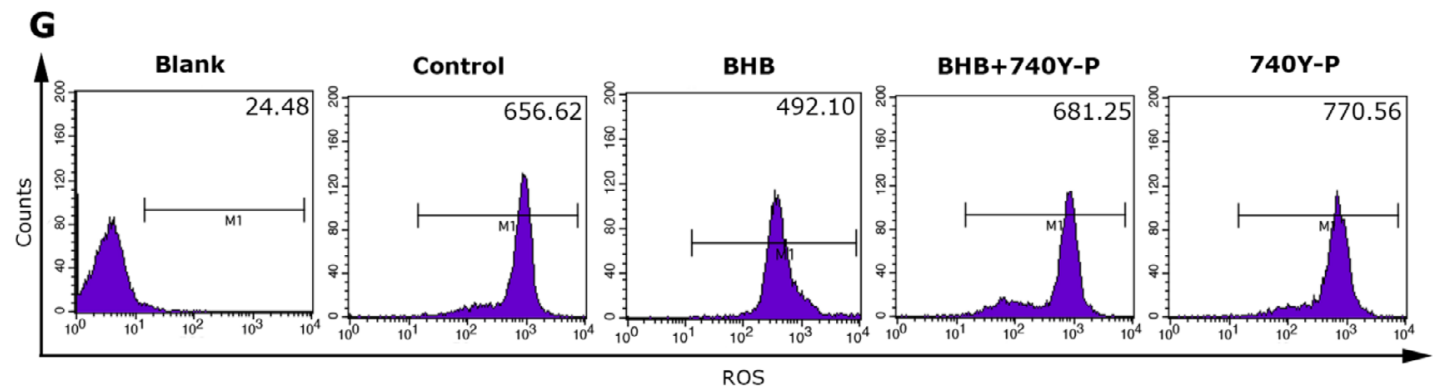

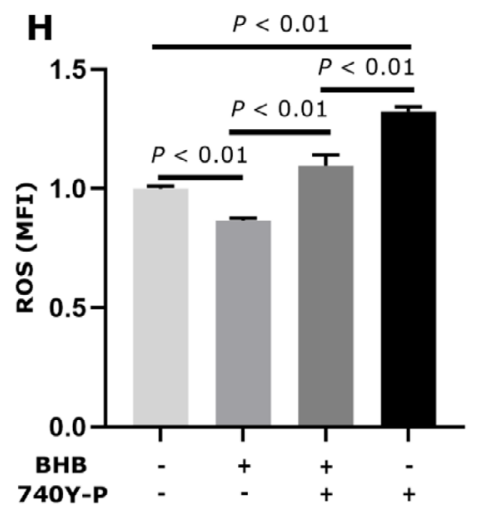

Figure 4. Role of the phosphoinositide 3-kinase (PI3K) signaling pathway in the BHB-induced inhibition of NADPH oxidase reactive oxygen species (ROS) production. (A, B) Protein abundance of PI3K and phosphorylated (p)-PI3K. Representative blots for both groups are shown in panel A, and the quantification in panel B. (C-F) Protein abundance of PI3K and p-PI3K, p47 and p-p47, and Rac2. 740Y-P, an activator of the PI3K signaling pathway, was preincubated for $0.5 \mathrm{~h}$ before the actual experiments. Representative blots for both groups are shown in panel $\mathrm{C}$, and the quantification in panel $\mathrm{D}-\mathrm{F}$. $\beta$-Actin was used to normalize the protein abundance. Two-way ANOVA revealed a BHB $\times 740 Y-\mathrm{P}$ interaction $(P<0.05)$. (G) Flow cytometry analysis of intracellular ROS. (H) Quantitation of ROS levels in panel G. Experiments were repeated 5 times. Two-way ANOVA revealed a BHB $\times 740 \mathrm{Y}$-P interaction $(P<0.05)$. Data were analyzed with 2 -way ANOVA or independent-sample $t$ tests. Data are expressed as means \pm SEM. A $P<0.05$ was deemed significant and $P<0.01$ highly significant. Flow cytometry analysis of intracellular ROS. PMA = phorbol-12-myristate-13-acetate; MFI = mean fluorescent intensity; M1 = fluorescence intensity of ROS-producing cells. 
A
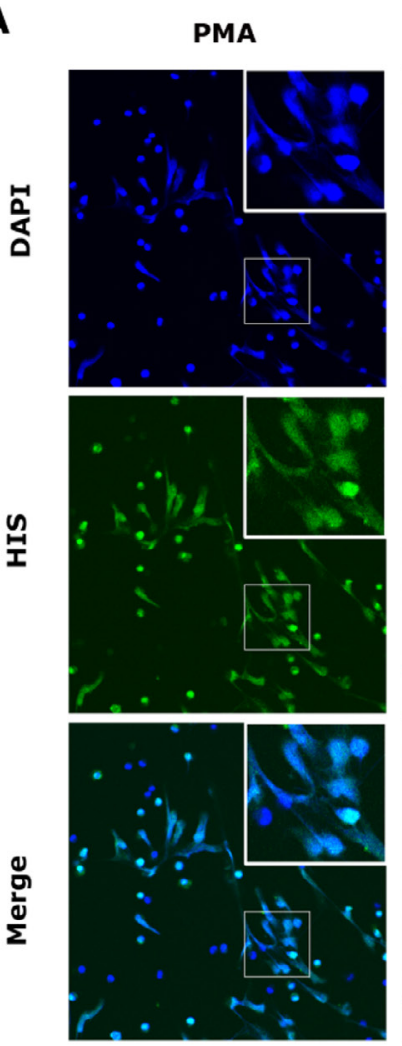

Ratio of NETs cells

(Mean \pm SEM
$1.000 \pm 0.037$
BHB+PMA
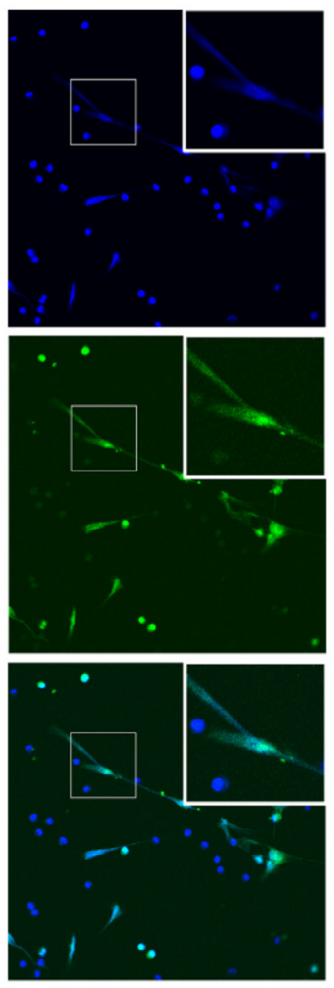

$0.515 \pm 0.012$
BHB+PMA

$+740 Y-P$
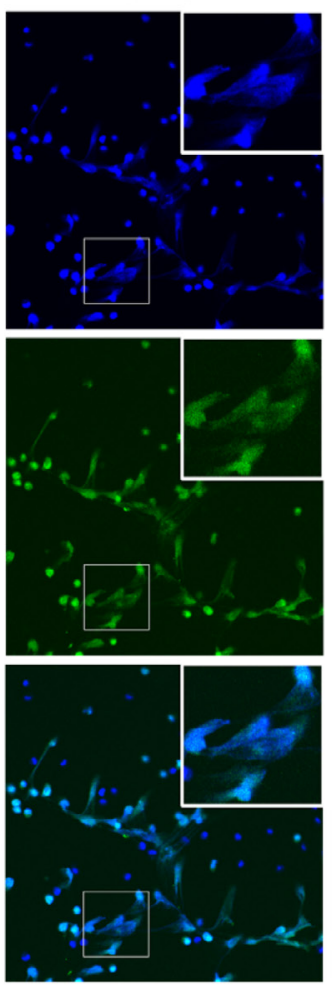

$0.951 \pm 0.025$
PMA+740Y-P
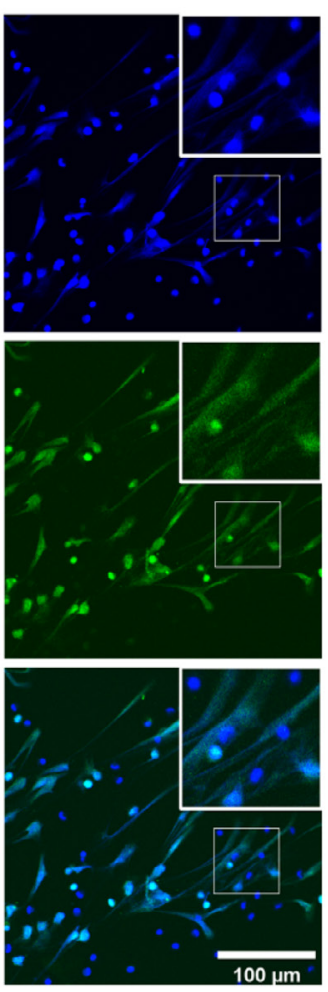

$1.218 \pm 0.017$

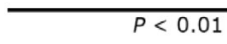

$P<0.01$

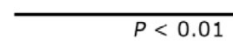

B

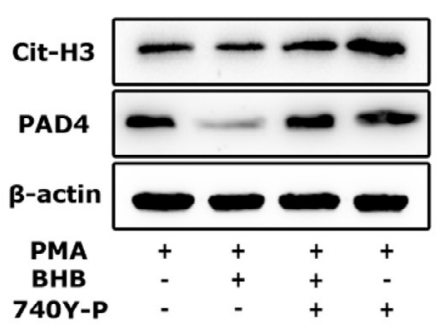

C

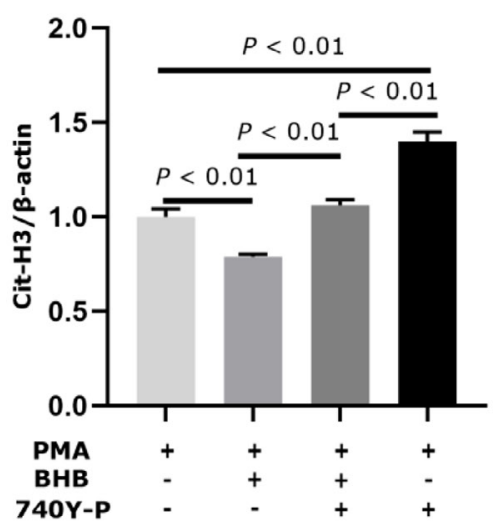

D

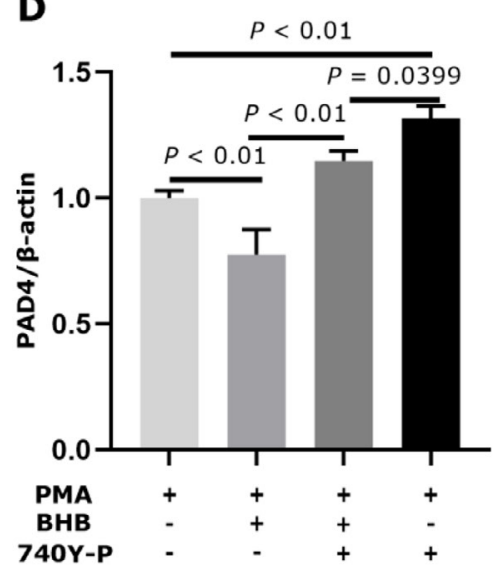

Figure 5. Role of the phosphoinositide 3-kinase (PI3K) signaling pathway in BHB inhibited release of bovine neutrophil extracellular traps (NET). The PMA (100 ng/mL) treatment for $3 \mathrm{~h}$ was used to induce NET formation. (A) Immunofluorescence experiments revealed release of NET structures from PMN. Magnification $=400 \times$. Scale bar $=100 \mu \mathrm{m}$. Nuclei/DAPI is in blue, HIS is in green. Images represent the typical common phenotype of each group. Ratio of NET cells are shown under the image. At least 500 cells were calculated in each group. Two-way ANOVA revealed a BHB $\times 740$ Y-P interaction $(P<0.01)$. (B-D) Protein abundance of Cit-H3 and PAD4. Representative blots for both groups are shown in panel $\mathrm{B}$, and the quantification in panel $\mathrm{C}$ and $\mathrm{D}$. $\beta$-Actin was used to normalized the protein abundance. Two-way ANOVA revealed a BHB $\times 740 \mathrm{Y}-\mathrm{P}$ interaction for Cit-H3 $(P<0.05)$. The above experiments were repeated 5 times. Data are expressed as means \pm SEM. A $P<0.05$ was deemed significant and $P<0.01$ highly significant. HIS $=$ histone H3; Cit-H3 = citrullinated histone 3 ; PAD4 $=$ protein arginine deiminase 4 . 
strated that BHB can inhibit NET formation induced by PMA in bovine PMN, suggesting that BHB might affect the release of NET by regulating an upstream signaling pathway. Available studies indicated that NET formed by bovine PMN play an important role in the innate immune response against pathogens, and impaired NET formation or function is likely associated with increased sensitivity to infectious diseases such as mastitis (Grinberg et al., 2008). During NEB, PMN function is impaired in peripartal dairy cows, causing persistent infection (Swain et al., 2015). Thus, BHBinduced inhibition of NET release may be one of the main causes of immune dysfunction in ketotic cows.

The formation of NET depends on production of ROS, and NADPH oxidase is the major source in phagocytes (Lam et al., 2010; Grob et al., 2020). Pathological concentrations of BHB impair ROS production in bovine PMN (Grinberg et al., 2008). Our results also demonstrated that clinical concentration of BHB impair ROS production in bovine PMN. The NADPH oxidase is a group of plasma membrane-associated enzymes found in a variety of cells of mesodermal origin (Babior, 1999). Activation of these enzymes is mediated by the phosphorylation of $\mathrm{p} 47$ and the combination of Rac2 to gp91, steps that are crucial for the formation of NET in human PMN (El-Benna et al., 2009; Kirchner et al., 2012). In agreement, the present study revealed that phosphorylation of $\mathrm{p} 47$, protein abundance of Rac2, and intracellular ROS in bovine PMN decreased significantly after BHB treatment. This suggested that BHB decreased NADPH oxidase-derived ROS, thereby resulting in inhibition of NET release in bovine PMN. We speculate that infiltration of PMN by circulating BHB during ketotic conditions in the long term might lead to the inhibition of NADPH oxidase activation. Thus, when pathogens enter the host, PMN are unable to produce enough ROS and lose the ability to produce NET. Such effects likely contribute to secondary infectious disease in ketotic cows.

It was also reported that activation of PI3K signaling was important to the release of ROS-dependent NET in carp PMN (Zheng et al., 2020). Clinical concentration of BHB inhibited the activation of PI3K signaling in bovine hepatocytes (Angeli et al., 2021). Thus, together, available evidence suggests that clinical concentration of BHB might impair NET formation through inhibiting the PI3K-NADPH oxidase-ROS signaling pathway. In the present study, this hypothesis was confirmed by the inhibition in the release of NET after BHB treatment as well as the activation of PI3K signaling with 740Y-P. Thus, a potential approach to help alleviate risks of BHB inhibition of NET formation in dairy cows could encompass the use of additives or drugs that can target/activate PI3K or NADPH oxidase.
In that context, the antioxidant resveratrol and oat $\beta$-Glucans were demonstrated to attenuate PI3K/Aktmediated oxidative stress in different cells including PMN (Murphy et al., 2007; Zhuang et al., 2019, 2021). Apigenin, a naturally occurring flavone in plants, was demonstrated to activate NADPH oxidase and increase ROS production in different cells including PMN (Choi et al., 2007). The above molecules targeting PI3K and NADPH oxidase could have potential practical value in regulating immune dysfunction during NEB or ketosis.

In conclusion, our data demonstrated that clinical concentration of BHB impair NET release through inhibiting the PI3K-NADPH oxidase-ROS signaling pathway. The data add mechanistic information in regard to immune dysfunction during periods of NEB and ketosis in dairy cows.

\section{ACKNOWLEDGMENTS}

This work was supported by the National Natural Science Foundation of China (Beijing, China; grant nos. U20A2062 and 32060818). The authors have not stated any conflicts of interest.

\section{REFERENCES}

Angeli, E., D. Barcarolo, L. Durante, G. Santiago, V. Matiller, F. Rey, H. H. Ortega, and G. J. Hein. 2021. Effect of precalving body condition score on insulin signaling and hepatic inflammatory state in grazing dairy cattle. Domest. Anim. Endocrinol. 76:106621. https: //doi.org/10.1016/j.domaniend.2021.106621.

Babior, B. M. 1999. NADPH oxidase: An update. Blood 93:1464-1476. https://doi.org/10.1182/blood.V93.5.1464.

Borregaard, N. 2010. Neutrophils, from marrow to microbes. Immunity 33:657-670. https://doi.org/10.1016/j.immuni.2010.11.011.

Bréchard, S., S. Plancon, and E. J. Tschirhart. 2013. New insights into the regulation of neutrophil NADPH oxidase activity in the phagosome: A focus on the role of lipid and $\mathrm{Ca} 2+$ signaling. Antioxid. Redox Signal. 18:661-676. https://doi.org/10.1089/ars.2012.4773.

Bucktrout, R. E., N. Ma, A. Aboragah, A. S. Alharthi, Y. Liang, V. Lopreiato, M. G. Lopes, E. Trevisi, I. A. Alhidary, C. Fernandez, and J. J. Loor. 2021. One-carbon, carnitine, and glutathione metabolism-related biomarkers in peripartal Holstein cows are altered by prepartal body condition. J. Dairy Sci. 104:3403-3417. https:/ /doi.org/10.3168/jds.2020-19402.

Burgener, S. S., and K. Schroder. 2020. Neutrophil extracellular traps in host defense. Cold Spring Harb. Perspect. Biol. 12:a037028. https://doi.org/10.1101/cshperspect.a037028.

Choi, S. I., C. S. Jeong, S. Y. Cho, and Y. S. Lee. 2007. Mechanism of apoptosis induced by apigenin in HepG2 human hepatoma cells: Involvement of reactive oxygen species generated by NADPH oxidase. Arch. Pharm. Res. 30:1328-1335. https://doi.org/10.1007/ BF02980274.

Dervishi, E., G. Zhang, D. Hailemariam, S. A. Goldansaz, Q. Deng, S. M. Dunn, and B. N. Ametaj. 2016. Alterations in innate immunity reactants and carbohydrate and lipid metabolism precede occurrence of metritis in transition dairy cows. Res. Vet. Sci. 104:30-39. https://doi.org/10.1016/j.rvsc.2015.11.004.

Du, X., T. Shen, H. Wang, X. Qin, D. Xing, Q. Ye, Z. Shi, Z. Fang, Y. Zhu, Y. Yang, Z. Peng, C. Zhao, B. Lv, X. Li, G. Liu, and X. Li. 2018a. Adaptations of hepatic lipid metabolism and mitochondria in dairy cows with mild fatty liver. J. Dairy Sci. 101:9544-9558. https://doi.org/10.3168/jds.2018-14546. 
Du, X., Y. Zhu, Z. Peng, Y. Cui, Q. Zhang, Z. Shi, Y. Guan, X. Sha, T. Shen, Y. Yang, X. Li, Z. Wang, X. Li, and G. Liu. 2018b. High concentrations of fatty acids and beta-hydroxybutyrate impair the growth hormone-mediated hepatic JAK2-STAT5 pathway in clinically ketotic cows. J. Dairy Sci. 101:3476-3487. https://doi.org/10 .3168/jds.2017-13234.

El-Benna, J., P. M. C. Dang, M. A. Gougerot-Pocidalo, J. C. Marie, and F. Braut-Boucher. 2009. p47phox, the phagocyte NADPH oxidase/NOX2 organizer: Structure, phosphorylation and implication in diseases. Exp. Mol. Med. 41:217-225. https://doi.org/10.3858/ emm.2009.41.4.058.

FASS. 2010. Guide for the Care and Use of Agricultural Animals in Research and Teaching. 3rd ed. FASS Inc.

Galvão, K. N., M. J. B. F. Flaminio, S. B. Brittin, R. Sper, M. Fraga, L. Caixeta, A. Ricci, C. L. Guard, W. R. Butler, and R. O. Gilbert. 2010. Association between uterine disease and indicators of neutrophil and systemic energy status in lactating Holstein cows. J. Dairy Sci. 93:2926-2937. https://doi.org/10.3168/jds.2009-2551.

Grinberg, N., S. Elazar, I. Rosenshine, and N. Y. Shpigel. 2008. Betahydroxybutyrate abrogates formation of bovine neutrophil extracellular traps and bactericidal activity against mammary pathogenic Escherichia coli. Infect. Immun. 76:2802-2807. https://doi .org/10.1128/IAI.00051-08.

Grob, D., I. Conejeros, Z. D. Velasquez, C. Preusser, U. Gartner, P. Alarcon, R. A. Burgos, C. Hermosilla, and A. Taubert. 2020. Trypanosoma brucei brucei induces polymorphonuclear neutrophil activation and neutrophil extracellular traps release. Front. Immunol. 11:559561. https://doi.org/10.3389/fimmu.2020.559561.

Itle, A. J., J. M. Huzzey, D. M. Weary, and M. A. von Keyserlingk. 2015. Clinical ketosis and standing behavior in transition cows. J. Dairy Sci. 98:128-134. https://doi.org/10.3168/jds.2014-7932.

Kirchner, T., S. Moller, M. Klinger, W. Solbach, T. Laskay, and M. Behnen. 2012. The impact of various reactive oxygen species on the formation of neutrophil extracellular traps. Mediators Inflamm. 2012:849136.

Lam, G. Y., J. Huang, and J. H. Brumell. 2010. The many roles of NOX2 NADPH oxidase-derived ROS in immunity. Semin. Immunopathol. 32:415-430. https://doi.org/10.1007/s00281-010-0221-0.

Lopes, M. G., A. S. Alharthi, V. Lopreiato, E. Abdel-Hamied, Y. Liang, D. N. Coleman, H. Dai, M. N. Correa, M. T. Socha, M. A. Ballou, E. Trevisi, and J. J. Loor. 2021. Maternal supplementation with cobalt sources, folic acid, and rumen-protected methionine and its effects on molecular and functional correlates of the immune system in neonatal Holstein calves. J. Dairy Sci. 104:93409354. https://doi.org/10.3168/jds.2020-19674.

Lopreiato, V., M. Vailati-Riboni, C. Parys, C. Fernandez, A. Minuti, and J. J. Loor. 2020. Methyl donor supply to heat stress-challenged polymorphonuclear leukocytes from lactating Holstein cows enhances 1-carbon metabolism, immune response, and cytopro- tective gene network abundance. J. Dairy Sci. 103:10477-10493. https://doi.org/10.3168/jds.2020-18638.

Murphy, E. A., J. M. Davis, A. S. Brown, M. D. Carmichael, A. Ghaffar, and E. P. Mayer. 2007. Oat beta-glucan effects on neutrophil respiratory burst activity following exercise. Med. Sci. Sports Exerc. 39:639-644. https://doi.org/10.1249/mss.0b013e3180306309.

Song, Y., N. Li, J. Gu, S. Fu, Z. Peng, C. Zhao, Y. Zhang, X. Li, Z. Wang, X. Li, and G. Liu. 2016. Beta-Hydroxybutyrate induces bovine hepatocyte apoptosis via an ROS-p38 signaling pathway. J. Dairy Sci. 99:9184-9198. https://doi.org/10.3168/jds.2016-11219.

Song, Y., J. J. Loor, C. Li, Y. Liang, N. Li, X. Shu, Y. Yang, X. Feng, X. Du, Z. Wang, G. Liu, and X. Li. 2021. Enhanced mitochondrial dysfunction and oxidative stress in the mammary gland of cows with clinical ketosis. J. Dairy Sci. 104:6909-6918. https://doi.org/ 10.3168/jds.2020-19964.

Song, Z., E. Hudik, R. Le Bars, B. Roux, P. M. Dang, J. El Benna, O. Nusse, and S. Dupre-Crochet. 2020. Class I phosphoinositide 3-kinases control sustained NADPH oxidase activation in adherent neutrophils. Biochem. Pharmacol. 178:114088. https://doi.org/10 .1016/j.bcp.2020.114088.

Swain, D. K., M. S. Kushwah, M. Kaur, and A. K. Dang. 2015. Neutrophil dynamics in the blood and milk of crossbred cows naturally infected with Staphylococcus aureus. Vet. World 8:336-345. https: //doi.org/10.14202/vetworld.2015.336-345.

Tackenberg, H., S. Moller, M. D. Filippi, and T. Laskay. 2021. The small GTPase Cdc42 negatively regulates the formation of neutrophil extracellular traps by engaging mitochondria. Front. Immunol. 12:564720. https://doi.org/10.3389/fimmu.2021.564720.

Wilson, R. M., and W. G. Reeves. 1986. Neutrophil phagocytosis and killing in insulin-dependent diabetes. Clin. Exp. Immunol. 63:478484.

Zhang, K. L., R. Dong, X. Z. Hu, C. Z. Ren, and Y. W. Li. 2021. Oat-based foods: Chemical constituents, glycemic index, and the effect of processing. Foods 10:1304. https://doi.org/10.3390/ foods10061304

Zheng, S., S. Wang, Q. Zhang, Z. Zhang, and S. Xu. 2020. Avermectin inhibits neutrophil extracellular traps release by activating PTEN demethylation to negatively regulate the PI3K-ERK pathway and reducing respiratory burst in carp. J. Hazard. Mater. 389:121885. https://doi.org/10.1016/j.jhazmat.2019.121885.

Zhu, Y., G. Liu, X. Du, Z. Shi, M. Jin, X. Sha, X. Li, Z. Wang, and X. Li. 2019. Expression patterns of hepatic genes involved in lipid metabolism in cows with subclinical or clinical ketosis. J. Dairy Sci. 102:1725-1735. https://doi.org/10.3168/jds.2018-14965.

Zhuang, Y., H. R. Wu, X. X. Wang, J. Y. He, S. P. He, and Y. L. Yin. 2019. Resveratrol attenuates oxidative stress-induced intestinal barrier injury through PI3K/Akt-mediated Nrf2 signaling pathway. Oxid. Med. Cell. Longev. 2019:7591840. https://doi.org/10 $.1155 / 2019 / 7591840$ 